\title{
Antimicrobial utilization: Capital Health Region, Alberta
}

Edith Blondel-Hill MD FRCPC ${ }^{1}$, Susan Fryters BScPharm ${ }^{2}$

$\mathrm{R}$ egionalization of health care services in Alberta began in 1994. In the Capital Health region, restructuring of seven hospitals resulted in two acute care hospitals, two community health centres, one rehabilitation hospital, one continuing care facility and the closure of one hospital. Reorganization of both pharmacy services and laboratory medicine have had a positive impact on antimicrobial utilization in the Capital Health region, which services Edmonton and surrounding areas. The major initiatives from the two disciplines are described below and summarized in Tables 1 and 2 .

\section{REGIONAL PHARMACY SERVICES}

Regional Committees: A regional Pharmacy and Therapeutics committee (P\&T) was formed in November 1995. Members are pharmacists, physicians, nurses and administration. The committee reports to the Regional Medical Directors Group.

Five drug utilization subcommittees were also formed, including an Antimicrobial Advisory Subcommittee (AASc). The subcommittees all report to the regional P\&T.

AASc members are physicians from adult and paediatric infectious diseases, medical microbiology, infection control, internal medicine, surgery, critical care and haematology/oncology; pharmacists with expertise in antimicrobial utilization, pharmacoeconomics and critical care; and administrative representatives with links to research and outcome, and continuous quality improvement.

The Regional Antimicrobial Working Group (AWG) is the workhorse of the AASc, and AWG members do all of the background work, including literature searches, audits and other research to bring issues to the AASc. One physician and five pharmacists are members.

The chair of the AASc and AWG is a medical microbiologist and infectious diseases physician, who works 0.5 full-time equivalent as an antimicrobial utilization specialist for the
TABLE 1

Antimicrobial utilization initiatives in the Capital Health region of Alberta

Regional committees

Pharmacy and Therapeutics

Antimicrobial Advisory Subcommittee

Antimicrobial Working Group

Regional antimicrobial formulary

Antibiotic request form

Automatic stop orders

Automatic therapeutic substitutions

Step down recommendations

Recommendations for antimicrobial surgical prophylaxis

Clinical guidelines for antibiotics

Recommendations for empirical therapy of selected infections

Antibiotic audits

Antimicrobial Pocket Reference

TABLE 2

Microbiology laboratory services' antimicrobial utilization intiatives in the Capital Health region of Alberta

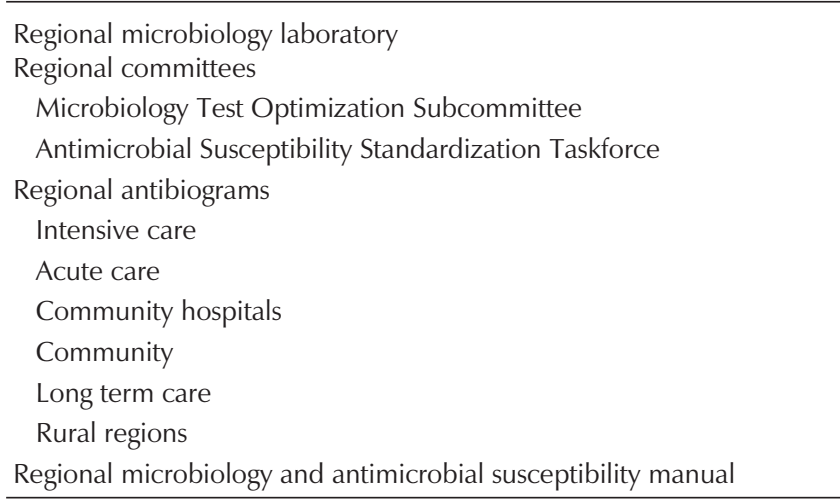

\footnotetext{
${ }^{1}$ Dynacare Kasper Medical Laboratory, Edmonton, Alberta; ${ }^{2}$ Capital Health Regional Pharmacy Services, Edmonton, Alberta

Correspondence: Dr E Blondel-Hill, Dynacare Kasper Medical Laboratory, 14940 - 123 Avenue NW, Edmonton, Alberta T5V 1B4. Telephone 403-451-3702, fax 403-452-8488, e-mail eblondel@cha.ab.ca
} 
Capital Health region, and 0.5 full-time equivalent as a medical microbiologist for Dynacare Kasper Medical Laboratories (DKML). The secretary of the AASc and AWG is a full-time antimicrobial utilization and infectious diseases pharmacist.

Regional antimicrobial formulary: The antimicrobial formularies of the individual hospitals were reviewed, and in January 1996, the AASc approved a single list of antibiotics that would be available for use within the Capital Health region. In addition to the benefits of standardizing this group of drugs, some cost savings were also realized. With no duplication within antibiotic classes, most antimicrobials were included on the regional formulary. However, use of many of the broad spectrum antibiotics, such as third-generation cephalosporins and imipenem, was restricted and requires the completion of an antibiotic request form.

Regional antibiotic form: Prescription of all restricted and nonformulary antimicrobials requires completion of the regional antibiotic form (Figure 1). The main purpose of this form is not to act as a deterrent to the prescription of restricted and nonformulary antibiotics but to act as a data collection tool for the pharmacists on the patient care units and the antibiotic utilization team to assess all aspects of the antibiotic therapy ordered. Data from these forms are compiled and retrospectively evaluated by antimicrobial utilization pharmacists using a standard grading scale. These monthly reports, combined with the corresponding expenditures, are then reviewed by the AWG and AASc. The ultimate goal is to report antimicrobial utilization and expenditure data regularly to each of the medical programs in order to optimize the use of these broad spectrum agents.

Automatic stop orders: All drugs ordered in the Capital Health hospital system have a standard automatic stop order (ASO). In general, systemic antimicrobials have an ASO of seven days. Azithromycin and antituberculous medications are exceptions, with an ASO of five and 30 days, respectively. ASOs help curtail unnecessarily long courses of antibiotic therapy and ensure regular review of existing antibiotic orders.

Automatic therapeutic substitutions: Similar to what was done with individual formularies, the AASc reviewed automatic therapeutic substitutions that were in place at each of the institutions and developed a single list of substitutions for the region. Hospital pharmacists have been authorized by the regional $P \& T$ to implement 28 substitutions. Some involve substitution of the most appropriate dose (eg, ceftriaxone $1 \mathrm{~g}$ daily except in meningitis or other central nervous system infections, or intravenous cefuroxime $750 \mathrm{mg}$ every $8 \mathrm{~h}$ ) or interval (eg, intravenous cefazolin and clindamycin every $8 \mathrm{~h}$ or intravenous metronidazole every $12 \mathrm{~h}$ ). Other automatic therapeutic substitutions entail the use of a different antibiotic, eg, ciprofloxacin instead of norfloxacin or imipenem instead of meropenem. Automatic substitutions are documented on the patient care record, and the prescribing physician always has the option of overriding the intervention if deemed necessary. Antibiotic substitutions are intended to take advantage of desirable pharmacokinetics, enforce the formulary with a resulting savings in time and money and maintain optimal antimicrobial therapy for the patient.

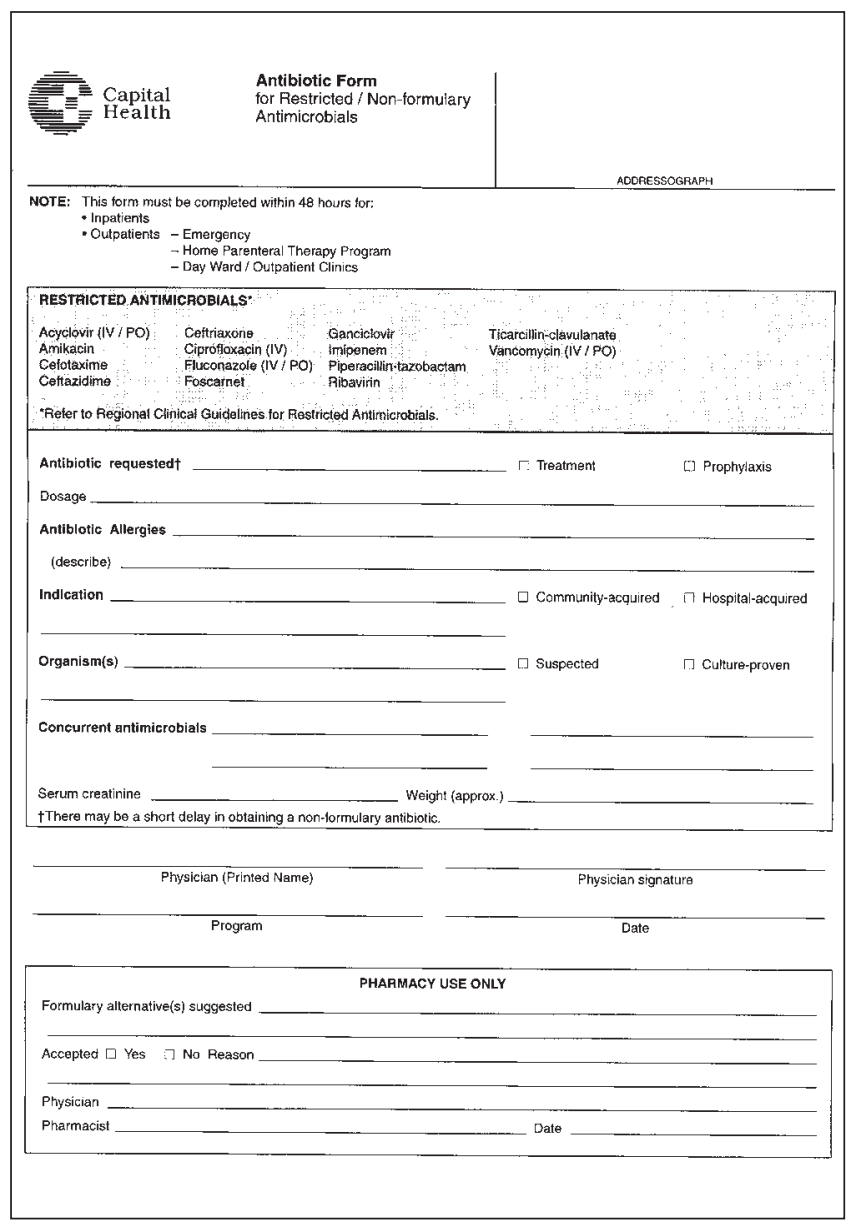

Figure 1) Capital Health regional antibiotic form for restricted and nonformulary antimicrobials

Step down recommendations: The AASc has provided regional recommendations for converting patients' antimicrobial therapy from the parenteral to the oral route. At this time, the following parenteral anti-infectives are included: ampicillin, cefazolin, cefuroxime, ciprofloxacin, clindamycin, fluconazole and metronidazole.

Recently, the automatic step down of intravenous ciprofloxacin has been implemented in the region. Pharmacists working on patient care units are authorized to convert ciprofloxacin to the oral route if the patient is tolerating oral medications, or enteral feeds and any potential drug interactions are resolved. This single conversion could save up to $\$ 70,000$ in the region.

Recommendations for antimicrobial surgical prophylaxis: Recommendations for appropriate antimicrobial prophylaxis were developed for all surgical procedures commonly performed in the Capital Health region. These list potential pathogens involved in wound infections, the prophylactic regimen of choice and alternative regimens for each procedure. A single preoperative dose was recommended for the majority of procedures. Also, vancomycin use was greatly limited, with clindamycin recommended in vancomycin's place for orthopedic and selected vascular procedures. Standardization of prophylaxis 
TABLE 3

Antimicrobial Pocket Reference table of contents

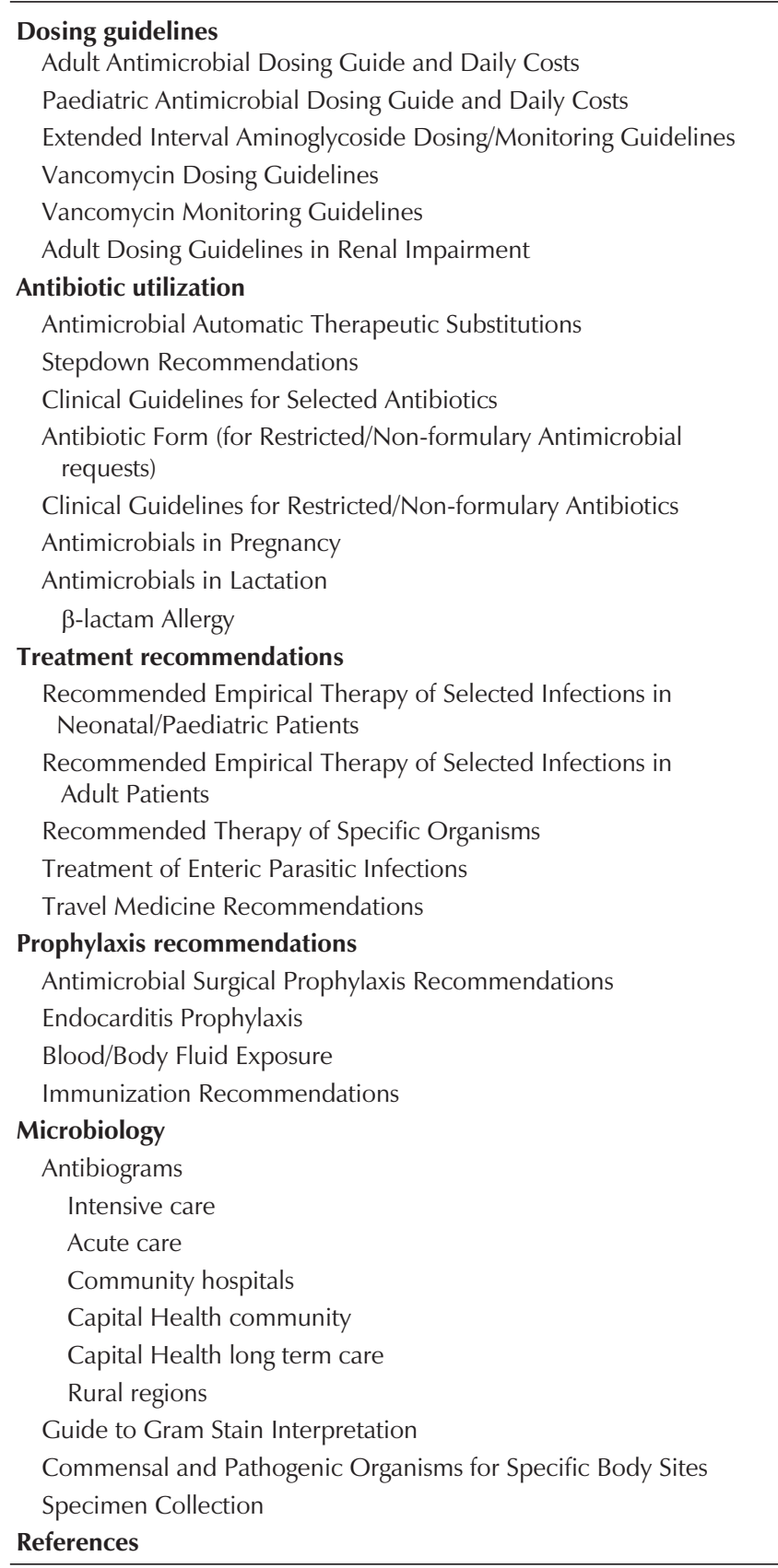

is important to prevent postsurgical infections, the use of broad spectrum agents and the development of resistance.

A regional policy for the administration of surgical prophylaxis was also implemented. Anesthetists administer the antibiotics just before surgery, thus optimizing the timing of administration.

Clinical guidelines for antibiotics: Guidelines for the appropriate use of the majority of oral antibiotics and all restricted agents were developed. Guidelines for several expensive and/or broad spectrum nonformulary agents, such as lipidcomplexed amphotericin $\mathrm{B}$, cefepime and meropenem, were also developed in a prospective effort to curtail their use. Infor- mation included in these guidelines are spectrum of activity, indications for use and indications where these agents should not be used.

Recommendations for empirical therapy of selected infections: Recommendations for the empirical therapy of most community- and hospital-acquired infections for both pediatric and adult patients were developed. Empirical regimens include common pathogens, and the appropriate antibiotic, dose and duration of therapy for each infection. Standardization of empirical therapy will help prevent antimicrobial resistance and contain costs, while optimizing the care of patients with infectious diseases.

Audits: Audits of antimicrobial use are another important component of the Capital Health region's antimicrobial utilization program. These audits have been done either retrospectively or prospectively, and have identified trends in antibiotic use in the region or documented the success of the various initiatives implemented by the antimicrobial utilization team. Audits can be drug- or disease-specific, or focus on antimicrobial use within a specific patient care area, such as intensive care.

Antimicrobial Pocket Reference: The purpose of the Antimicrobial Pocket Reference is to standardize the treatment and prophylaxis of infections in order to rationalize antibiotic use and control the development of resistant organisms. The book addresses both community- and hospital-acquired infections, and includes most of the information described in this article. The complete table of contents can be found in Table 3 . This educatinal reference was prepared by the authors (EBH and SF) and reviewed by Adult and Paediatric Infectious Diseases, Medical Microbiology, Pharmacy and the AASc. Preparation and publication of the book was jointly supported and funded by the capital health region's pharmacy services and DKML. It was distributed to all physicians (including residents, fellows and interns), pharmacists and infection control practitioners in both the community and hospital settings in the Capital Health region, as well as in the surrounding regions serviced by DKML.

\section{REGIONAL MICROBIOLOGY LABORATORY SERVICES}

Regionalization of microbiology services involved the merging of three public and three private sector laboratories. DKML is now responsible for the delivery of microbiology services to the Capital Health region, excluding the University of Alberta Hospital, which retained its own microbiology laboratory. In addition, DKML provides microbiology services (consultative or managerial services, or full delivery of services) to most regions in northern and central Alberta, as well as to parts of the Northwest Territories.

Regional committees: A Microbiology Test Optimization Subcommittee was formed to standardize procedures and protocols within microbiology laboratories. Members are medical microbiologists, senior technologists, and managers from DKML, the University of Alberta Hospital's microbiology laboratory and the Provincial Laboratory of northern Alberta. The subcommittee reports to the Capital Health region's Test Optimization Committee for laboratory medicine. 
Susceptibility testing: Within the Capital Health region, a taskforce was formed to standardize susceptibility testing and reporting between DKML and the University of Alberta Hospital's microbiology laboratory. Susceptibility testing in the region is reported in a cascading fashion and in accordance with the antibiotics on the regional formulary.

Antibiograms from acute care, long term care and the community, highlighting differences in susceptibility patterns and antimicrobial resistance among the various sectors, were distributed to physicians and pharmacists as a part of the pocket reference.

Rural microbiology services: As part of the consultative serv- ice to various regions within Alberta and the Northwest Territories, a regional DKML microbiology manual was distributed and implemented at most regional microbiology laboratories. The result has been a standardized approach to the work-up of microbiology specimens and the reporting of susceptibility results.

In summary, regionalization has provided an opportunity to standardize and optimize the delivery of health care services. Antimicrobial use initiatives from both regional pharmacy and microbiology services in the Capital Health region of Alberta have allowed the growing problem of antimicrobial resistance to be confronted. 


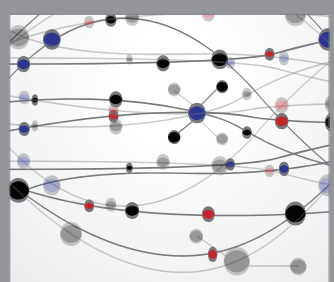

The Scientific World Journal
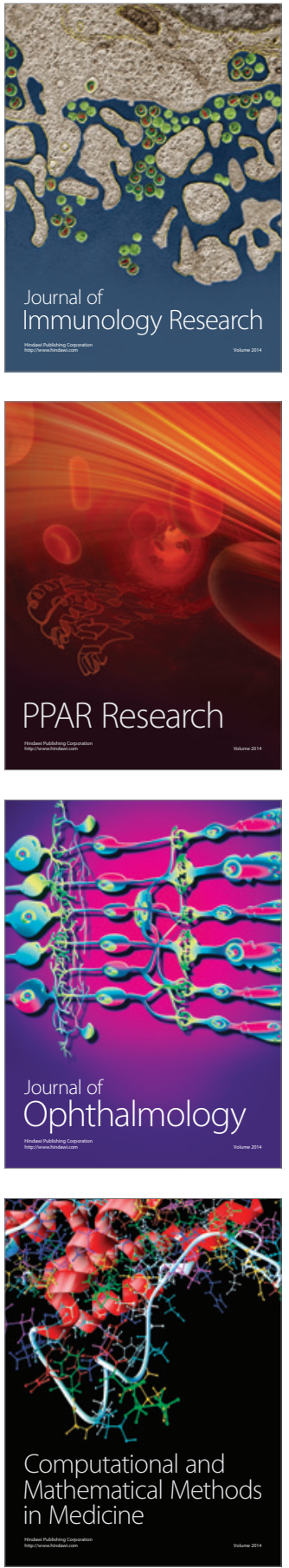

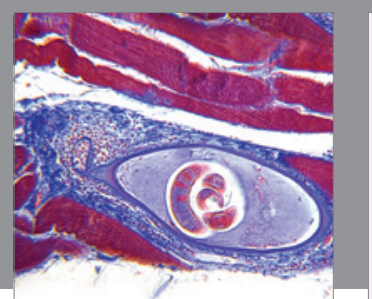

Gastroenterology Research and Practice

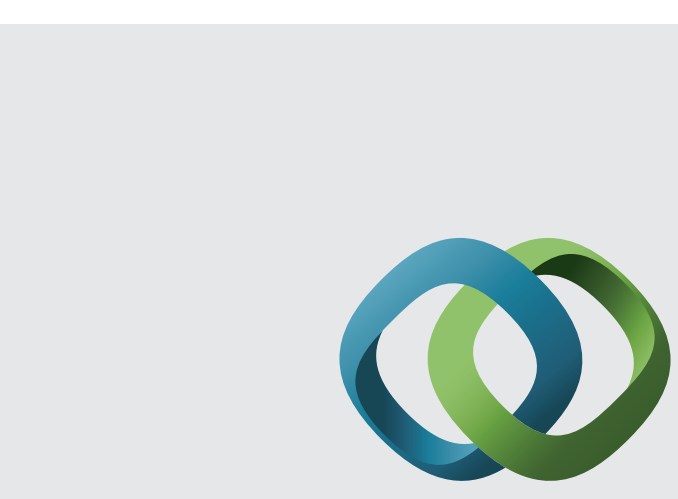

\section{Hindawi}

Submit your manuscripts at

http://www.hindawi.com
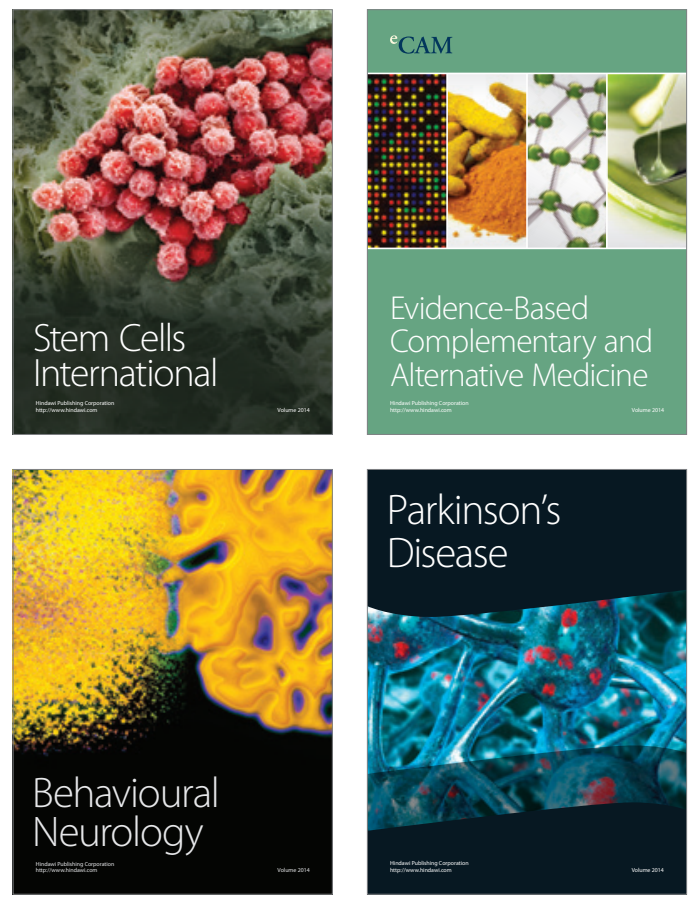
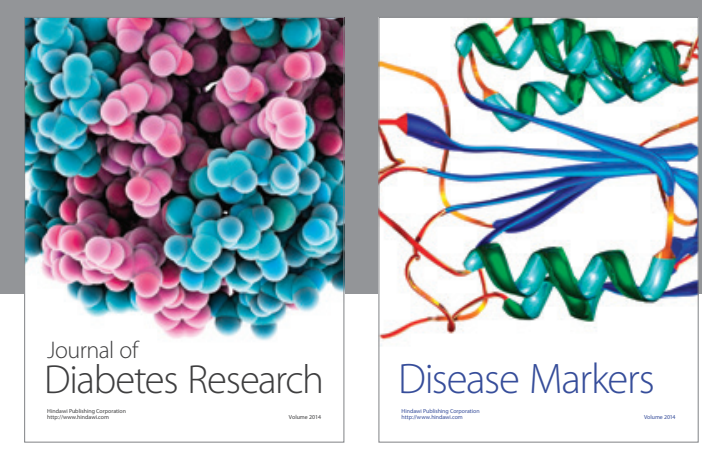

Disease Markers
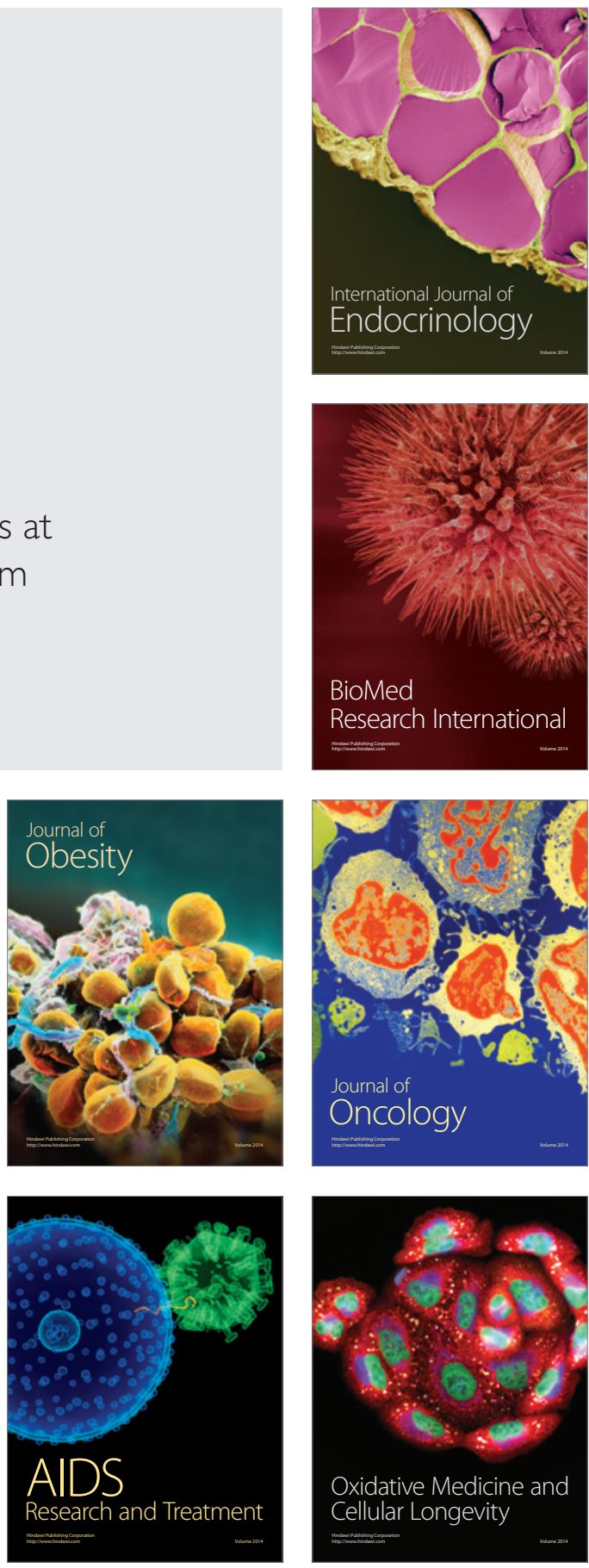